\title{
Redundancy Control Energy Efficient Clustering (RCEEC) protocol for Wireless Sensor Networks
}

\author{
Abhinav Garg \\ Associate Professor, National Institute of Fashion Technology, Hyderabad India \\ abhinavarungarg1@gmail.com \\ Dr. Manisha Jailia \\ Associate Professor, Banasthali Vidyapith, Rajasthan India \\ manishajailia@yahoo.co.in
}

\begin{abstract}
Wireless Sensor Networks(WSNs) refer to a collection of resource constrained, miniature sensor devices which can be embedded in places ranging from remote military areas, underwater research explorations to much needed everyday objects like doors, mirror, watch, apparels etc. Many heterogeneous clustering protocols have been studied so far for WSNs, but they concentrate on improving energy, latency and network lifetime. Here a Redundancy Control Energy Efficient Clustering (RCEEC) protocol for WSNs has been suggested which emphasizes on reducing irrelevant data from the network. Results show that RCEEC surpasses its predecessor DEEC with respect to energy efficiency, intra cluster traffic and network lifetime.
\end{abstract}

Keywords: Heterogeneous networks, Wireless Sensor Networks (WSN), Energy Efficiency, Clustering, Network Lifetime, Redundancy.

\section{Introduction}

Wireless Sensor Networks incorporates miniature sensing devices placed in the sensing zone to record the phenomenon like temperature, pressure, humidity, motion, light etc. Today the world around us is full of IOT devices that collect GB and TB of data every second. The Internet of Things (IoT) is a buzzword in every place around us, be it surveillance, urban monitoring, home management, security, medicine, travel, transport, engineering and much more. A network of multitudes of sensors and actuators stationed to sense the physical phenomenon and direct the data back to a Base Station (BS) or cloud is called a Wireless Sensor Networks. The backbone of any WSN is innumerous tiny IoT devices working behind the scenes [1]. They have limited battery power which is often irreplaceable and non- rechargeable. A node in a typical sensor network senses the environment, gathers the entire data and sends it to the sink for processing. At the Base Station data is consolidated and sent to the cloud servers. These servers serve as the data warehouse of knowledge discovery. This generates a voluminous amount of big data that becomes difficult to address and handle. Bulk of this data is highly redundant and of no use as the sensing zones of the nodes overlaps with each other. Sending the same type of data repeatedly, drains the energy of nodes unnecessarily which can otherwise be utilized to enhance the life of the network.

Much of the work in the development of sensor Networks have focused on saving energy to increase the longevity of networks, as replacement of batteries in sensing devices is a tedious task. The most commonly used approach for saving energy is clustering. Nodes are grouped into clusters. Every cluster decides on a Cluster Head $(\mathrm{CH})$ which takes the information from its associated nodes, process the information and relay it to the base station (BS) or another $(\mathrm{CH})$ which then forwards it along the shortest path to BS.A network can be classified as homogeneous or heterogeneous. A homogeneous network is one which is made up of nodes having similar energy levels. A heterogeneous network has different energy levels for the nodes.

The idea was first conceptualized by W. Heinzelmal et. al. in their protocol named LEACH [2]. LEACH gave the idea of dividing the entire cycle of network formation and data transmission in two stages: - Setup state and steady state. Set up state is responsible for handshake i.e. cluster formation whereas actual data transmission takes place in steady phase. Some nodes in heterogeneous settings having lesser energy levels are known as Normal Nodes. And the nodes which possess slightly higher energy and processing capabilities are called advanced nodes. All the nodes have the possibility to be elected as Cluster Heads $(\mathrm{CH})$. This heterogeneity was introduced to increase network lifetime. There is a random rotation of $\mathrm{CH}$ position to avoid battery drainage in any particular node. The protocol ensures that each node gets a fair chance to become a cluster head depending on probability (p). 
There are hundreds and thousands of nodes in a dense WSN. In IoT networks, sensors might be closely deployed with a high rate of overlapping sensing zones. Sensors record data at the resolution of milliseconds. The samples taken by sensors in the common spatial region record similar types of data. Thus wasting the resources like energy and bandwidth. To reduce the huge amount of data, many data compression and encoding schemes have been proposed by various authors. These approaches aim at reducing the voluminous data at the cloud or sink. However a cutting edge solution is required to address this issue from edge to cloud.

Many clustering protocols have been studied so far like SEP [3], DEEC [4], EDEEC [5], iE-DEEC [6], DDEEC [7]. All the above approaches concentrate on reducing the amount of energy spent in the network to increase the lifespan of the network. As suggested in [8] protocols like SIP [9], Bare Necessities [10], SAR [11] reduce redundancy in the network by following a sleep/awake schedule using TDMA. Though it reduces network traffic but at the cost of overhead of toggling between the sensor states and moreover in an edge enabled wireless sensor network, nodes might act independently also.

In-network redundancy can be addressed in two ways:

1) By reducing the size of data packets

2) By reducing the number of transmissions.

First solution is not highly yielding as the data packet in the sensor network is generally of low order of magnitude [12]. Major issue is to deal with the volumes of these packets generated every few seconds.

This paper addresses the above mentioned concern and the proposed approach focuses on minimizing the bulk of data being transmitted from individual nodes to the Cluster Heads (CHs). It takes into account the possibility of retransmissions of the same data due to overlapping regions or due to densely deployed nodes in the region.

Redundancy Control Energy Efficient Clustering (RCEEC) protocol works on the idea of minimizing the number of packets in transit to control the redundancy in the network. It has been successful in reducing the intra cluster communication by a significant amount. Simulation results show that energy dissipated per round decreases thus achieving better performance in terms of network lifetime.

\section{Literature Review}

Various data gathering, data dissemination, data aggregation, routing and scheduling algorithms for Wireless sensor networks (WSNs) have been studied extensively to make them energy efficient and improve the Quality of Service (QoS). Here we focus on clustering and data aggregation protocols as this proposed work has evolved from the same.

W. Heinzelman et. al.[13] developed a protocol, low-energy adaptive clustering hierarchy (LEACH). LEACH introduced a distributed, self-organizing cluster formation technique where new cluster heads are selected in every round to evenly distribute the network load. It ensures that each node gets a fair chance to become a cluster head depending on a probability (p).

$$
T(n)= \begin{cases}\frac{p}{1-p \bmod \left(r, \frac{1}{p}\right)} & \text { if } n \in G \\ 0 & \text { otherwise }\end{cases}
$$

Where $\mathrm{p}$ is Optimum Election Probability with which a node could be chosen as a cluster head, $r$ is the index of ongoing round, $\mathrm{N}$ is the number of nodes, $\mathrm{G}$ is the collection of nodes which are eligible for being a cluster head in that round. $\mathrm{CHs}$ take input from all the normal nodes, aggregates and forward it to the base station (BS). However as mentioned in [3], LEACH becomes unstable with the increase in heterogeneity.

Farooq et al. [14] have suggested an improvement over LEACH and named it as MR-LEACH (Multi-hop Routing with Low Energy Adaptive Clustering Hierarchy). They have split the network area into four concentric rings to minimize the distance between any $\mathrm{CH}$ of inner ring and its corresponding $\mathrm{CH}$ in outer level ring. It ensures that there is equal number of clustering levels for all the nodes. The results show that MR-Leach performs well and residual energy in the nodes is more than that in LEACH.

K. Maraiya et. al. [15] has recommended a data aggregation algorithm for wireless sensor network called efficient cluster head selection scheme for data aggregation (ECHSSDA). In this algorithm, an associate Cluster Head is chosen along with a normal Cluster Head. The associate $\mathrm{CH}$ node can replace the actual $\mathrm{CH}$ node when it stops functioning. The central idea is taken from a localized clustering protocol LEACH (Low Energy Adaptive Clustering Hierarchy). 
SEP [3] recommended by G. Smaragdakis et. al. is a heterogeneous network protocol where all the nodes have different energy levels. CHs are selected on the grounds of weighted election probabilities of individual node and the remaining energy of individual node.

$$
\begin{aligned}
& \text { No. of advanced nodes }=m * n \\
& \quad \text { No. of normal nodes }=(1-m) * n
\end{aligned}
$$

Where $\mathrm{n}$ denotes the total number of nodes and $\mathrm{m}$ represents the percentage of nodes which possess $\alpha$ times extra energy than the others.

$$
T_{\text {total }}=n \cdot E_{o}(1+\alpha \cdot m)
$$

Hence, there is an increase in the overall energy of the network by $1+\alpha \cdot \mathrm{m}$.

S. Kumar et al. recommended an energy aware reactive routing protocol with 3 levels heterogeneous setting, Enhanced Threshold Sensitive Stable Election Protocol for Heterogeneous Wireless Sensor Network ETSSEP [16]. The election probabilities rely on the remaining energy of node and aggregate energy of the network at round $\mathrm{r}$. With this there is an increase in the network lifetime and stability period.

S. Kumar et al. extended the previous SEP based protocols and proposed an energy aware reactive routing protocol with 3 different categories of nodes -. Advance nodes, intermediate nodes and normal nodes. Protocol was named as Enhanced Threshold Sensitive Stable Election Protocol for Heterogeneous Wireless Sensor Network ETSSEP [17]. The election probabilities rely on the remaining node energy and aggregate network energy in round $\mathrm{r}$.

To become a cluster head, the threshold for a node is calculated as:-

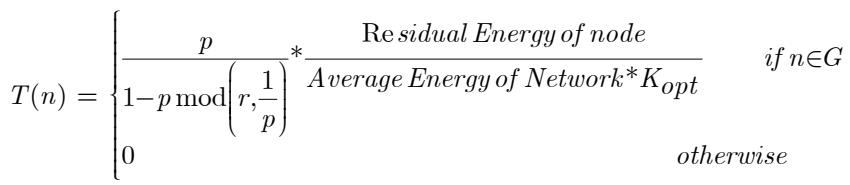

Authors claim that nodes with the highest energy will always be the preferred candidate for the cluster head.

In DEEC [4], the ideology of LEACH and HEED is comprehended. It works on multiple heterogeneous networks setting where all the nodes have different energy levels. A fixed number of $\mathrm{CHs}$ are elected per round depending on the probability of ratio of remaining energy and aggregate network energy. The authors claim to have achieved a uniform dispensation of energy enervation amongst the nodes, but it is observed that there is a high variation in no. of $\mathrm{CH}$ nodes per round and node degree of CHs. The results depict that DEEC outperforms LEACH and SEP. Therefore for this paper DEEC has been taken as a benchmark for comparing results.

Versions of DEEC like E-DEEC (Enhanced Distributed Energy Efficient Clustering) [5] and het-DEEC: heterogeneous distributed energy efficient clustering protocol [18] takes the ap-proach further and implement the network with three-level heterogeneity using weighted election probability. The authors claim to ameliorate the energy levels and the network lifetime.

Arati Manjeshwar et al. introduced a Threshold sensitive Energy Efficient sensor Network protocol TEEN [19] for networks which permit intermittent deviations in the pertinent parameters of the network like threshold. The idea was to have two thresholds Hard Threshold (HT) and Soft Threshold (ST). A node transmits data provided that the sensed value SV $>=$ HT and Current (SV) - Last (SV) $>=$ ST. After every round values of ST and HT can be adjusted to have control over the tradeoff between energy efficiency and accuracy. This approach however suffers a problem of lack of communication amongst nodes if thresholds are never reached.

Arati Manjeshwar and D. Agrawal propose Adaptive Threshold-sensitive Energy Efficient Network (APTEEN) [20], to overcome the loopholes in TEEN. CHs are responsible for sharing necessary parameters like threshold, TDMA schedule and a time counter (for controlling collisions). However, because of overburdening of CH's, they die prematurely.

J. Ma and S. Wang distributed the load of CH's in Adaptive Threshold-sensitive Energy Efficient Network based on ant colony (ADCAPTEEN) [21] and Multiple Adaptive Threshold-sensitive Energy Efficient Network based on Ant-colony (AMAP-TEEN) [22]. The authors suggest using two CHs viz. Master cluster head (MCH) and Vice cluster head $(\mathrm{VCH})$ per cluster that will work simultaneously on data collection, fusion and transmission. $\mathrm{MCH}$ collects the data from all the cluster members as per their TDMA schedule, processes the data and elects the member node that possesses the highest pheromone collection as Vice Cluster Head. Vice Cluster Head then communicates directly with Base Station. 
The pheromone concentration is calculated as:-

$$
\varsigma_{m i}=\frac{Q}{d_{m i}} * \frac{E_{m}}{E_{\text {init }}}+\beta * \frac{E_{i}}{E_{\text {init }}}
$$

Where $E_{i}$ represents the present energy of node i. $Q$ is a preset parameter. $E_{\text {init }}$ represents the energy of node $i$ at the beginning. $\beta$ is the adjusting factor. $d_{m i}$ represents the distance between a node $i$ and its corresponding Master Cluster Head.

In comparison to APTEEN [20], ADCAPTEEN [21] and AMAPTEEN [22] shows improved node survival rate, less energy loss and good scalability but still it suffers from a lot of overhead in the election process.

The work seen so far in literature focuses on optimizing energy levels and enhancing network lifetime. The size of clusters in the above protocols varies abruptly as they all follow a probabilistic approach to find the candidate $\mathrm{CHs}$ in a round. The count of normal nodes serviced by one $\mathrm{CH}$ (node degree of $\mathrm{CH}$ ) is also very uncertain. This leads to biased sampling of data, where one cluster might be receiving correlated data from its neighbors whereas other $\mathrm{CH}$ might be depending on a very few. Addressing one issue in these schemes compromise the other attributes like complexity, time taken and redundancy.

Here a simple technique has been proposed for a hierarchically distributed clustered network which is based on DEEC and its subsequent approaches.

\section{Radio Energy Dissipation Model}

Though various radio models exist in literature, this work focusses on the basic first order radio model.

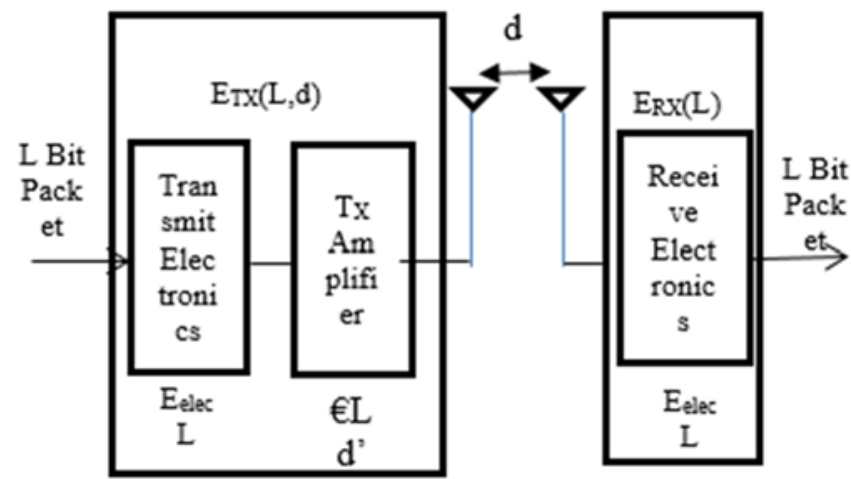

Figure1: Radio Energy Dissipation Model

Energy consumed by transmitter in sending L-bits of data across a distance d, by the radio is given by::-

$$
E_{T X}(L, d)= \begin{cases}L \cdot E_{\text {elec }}+L \cdot E_{f s} \cdot d^{2} & \text { if } d<d_{0} \\ L \cdot E_{\text {elec }}+L \cdot E_{m p} \cdot d^{4} & \text { if } d \geq d_{0}\end{cases}
$$

where $E_{\text {elec }}$ represents the energy consumed in every bit by the receiver circuit $\left(E_{\mathrm{rx}}\right)$ or the transmitter $\left(\mathrm{E}_{\mathrm{tx}}\right)$. $\mathrm{E}_{\text {elec }}$ is based on device specific features such as the digital coding, the modulation, the filtering, and the spreading of the signal [7]. For the experiments described here, the transmitter amplifier model uses the free space $\mathrm{E}_{\mathrm{fs}}$ with $\mathrm{d}^{2}$ power loss as well as the multipath fading $\mathrm{E}_{\mathrm{mp}}$ with $\mathrm{d}^{4}$ power loss models.

Threshold for distance amongst the transmitter and the receiver is calculated as:-

$$
d_{o}=\sqrt{\frac{E_{f s}}{E_{m p}}} \ldots \ldots . .(2)
$$

In case the distance (d) is smaller than $d_{0}$, the free space (fs) model ( $d^{2}$ power loss) is applied. If the distance (d) is greater than equal to do the multi path $(\mathrm{mp})$ model $\left(\mathrm{d}^{4}\right.$ power loss) is applied. Energy consumed by receiver can be expressed as:-

$$
E_{R X}=\left\{\begin{array}{ll}
L . E_{\text {elec }} & \text { for norma } 1 \text { nodes } \\
L .\left(E_{\text {elec }}+E_{D A}\right) & \text { for } C H^{\prime} s
\end{array} \ldots\right.
$$

where $\mathrm{E}_{\mathrm{DA}}$ is data aggregation energy. 


\section{Proposed Work}

Data Redundancy underlying in a huge sensor network is the root cause of failure and breakdown of sensor networks. Here, in this research, a new technique is suggested to minimize the amount of data being transmitted from individual nodes to the Cluster Heads. This approach, (RCEEC) Redundancy Control Energy Efficient Clustering protocol for WSNs works on the idea of minimizing the number of packets in transit to control the redundancy in the network.

Steps:

1. Set up a random network.

2. Preset energy parameters

3. Elect Cluster Head $(\mathrm{CH})$ based on optimal Probability

4. Associate nodes with the respective $\mathrm{CH}$

5. Transmit if and only if data packets received By cluster so far is less than equal to the Threshold determined

by the ratio of alive Nodes to the total number of clusters in round $r$

cluster.dp $<=\mathrm{T}(\mathrm{r})$

6. $\quad \mathrm{CH}$ aggregates and sends data to BS.

RCEEC considers the fact that the node degree (Number of nodes associated with $\mathrm{CH}$ at a time in a round) of clusters varies abruptly. In case of clusters with higher node degree, all the associated nodes need not send data to the cluster head, as the sensed data in a particular sensing zone will more or less be same. So only a fraction of alive nodes per cluster needs to transmit the data to $\mathrm{CH}$.

\subsection{System Architecture}

In the experiment, 100 nodes are arbitrarily placed in an area of $100 \times 100$. A Base station (BS) or sink is stationed at the middle of the plot. Sensor nodes are spatially dispersed and deterministically deployed on the field.

Simulation parameters and other radio characteristics are given in table (1).

Table 1- Simulation parameters

\begin{tabular}{|l|l|}
\hline Parameter & Value \\
\hline $\mathrm{N}$ & 100 \\
\hline $\mathrm{x}_{\mathrm{m}} \times \mathrm{y}_{\mathrm{m}}$ & $100 \mathrm{x} 100$ \\
\hline $\mathrm{E}_{\text {elec }}$ & $5.0 \mathrm{~nJ} / \mathrm{bit}$ \\
\hline $\mathrm{E}_{\mathrm{fx}}$ & $10.0 \mathrm{pJ} / \mathrm{bit} / \mathrm{m}^{2}$ \\
\hline $\mathrm{E}_{\mathrm{mp}}$ & $0.00130 \mathrm{pJ} / \mathrm{bit} / \mathrm{m}^{4}$ \\
\hline $\mathrm{E}_{0}$ & $0.50 \mathrm{~J}$ \\
\hline $\mathrm{E}_{\mathrm{DA}}$ & $5.0 \mathrm{~nJ} / \mathrm{bit} / \mathrm{message}$ \\
\hline $\mathrm{P}$ & 0.1 \\
\hline $\mathrm{R}_{\max }$ & 3000 \\
\hline $\mathrm{A}$ & 1 \\
\hline Do & 87.70 \\
\hline $\mathrm{L}$ & $4000 \mathrm{bits}$ \\
\hline
\end{tabular}

As proposed by DEEC [4] and its variants [5],[18] The network has multiple levels of heterogeneity where initial energy levels of nodes vary from Eo to $\mathrm{Eo}\left(1+\mathrm{a}_{\max }\right)$. Complete energy of the network initially is given by:

$$
E_{t}=\sum_{i=1}^{n} E_{o}\left(1+a_{i}\right)
$$

From eqn [4] it is clear that the nodes have definitely more energy level and better processing capabilities. This heterogeneity of nodes increases the capacity of the whole sensor network. 


\subsection{Cluster Head (CH) Selection}

Every node nominates itself for becoming a cluster head based on the average energy of the whole network. As in DEEC, every node gets a chance to be a $\mathrm{CH}$ once every $1 / \mathrm{p}$ round.

A node can be elected as a $\mathrm{CH}$ on the basis of the probability $\mathrm{p}_{\mathrm{i}}$

$$
p_{i}=\frac{p * n *(1+a) E_{i}}{\left(n+\sum_{i=1}^{n} a_{i}\right) * E_{a}} \ldots \ldots
$$

Where $E_{i}$ represents the current node energy and aggregate energy $E_{a}$ of the network in round $r$ is

$$
E_{a}=\frac{E_{t}\left(1-\frac{r}{r_{\max }}\right)}{n} \ldots . .
$$

Using eqn [5] Probability threshold can be calculated as given by [Leach, HEED, SEP, DEEC etc.]

$$
T(n)=\left\{\begin{array}{ll}
\frac{p}{1-p \bmod \left(r, \frac{1}{p}\right)} & \text { if } n \in G \\
0 & \text { otherwise }
\end{array} .\right.
$$

Each node generates an arbitrary number Temp and is elected as $\mathrm{CH}$ if

$$
\text { Temp }<=\text { Ti }
$$

$\mathrm{CHs}$ are accountable for collecting, summarizing and sending information to the BS. Energy dissipation is calculated as explained above in the first order radio model.

\subsection{Association of Nodes with $\mathrm{CH}$}

In this phase all the normal nodes determine either they have to communicate directly with BS or they would attach themselves to nearest $\mathrm{CH}$ (based on the Euclidean distances between node, $\mathrm{CH}$ and BS). All the nodes that decide to associate with the $\mathrm{CH}$, relay their data to $\mathrm{CH}$. However, a large amount of this data is highly repetitive. As sensors record and send data at an extremely high rate, all the data sent is not of much importance.

In the proposed approach (RCEEC), the redundant data relayed by the nodes to the respective CHs is discarded. The communication between a normal node $\mathrm{i}$ and respective $\mathrm{CH}$ is directly proportional to the average number of alive nodes per cluster. It is given by ratio of alive nodes in a certain round to the total number of clusters in that round.

So threshold for normal node to transmit data to $\mathrm{CH}$ becomes becomes:-

$$
T_{i->C H(i)}(r)=\varsigma * \frac{\operatorname{alive}(r)}{\text { cluster }(r)} \ldots \ldots . . .(9)
$$

$\tau$ is a redundancy control parameter whose value can be varied from .5 to 1 . We set $\tau$ as .6 which means that only $60 \%$ of the alive nodes in a cluster at a point of time in round $\mathrm{r}$ can participate in communication. Here cluster (r) refers to the number of clusters in round $\mathrm{r}$, alive (r) refers to number of alive nodes after round $\mathrm{r}$.

\section{Simulation Results}

This part shows the simulation results of both the DEEC and newly proposed REEC on the same environmental and network setting. We have evaluated the performance of both the protocols on Matlab software in terms of dead nodes, alive nodes, number of data packets relayed to CHs in every round.

General observations are summarized as under:

Table 2: Comparison between DEEC and RCEEC

\begin{tabular}{|l|l|l|}
\hline Parameter & DEEC & RCEEC \\
\hline Total dead nodes in end & 100 & 86 \\
\hline Round in which first node dies & 1201 & 1536 \\
\hline Round in which Tenth node dies & 1390 & 1786 \\
\hline Total no. of packets to CH & 130299 & 119870 \\
\hline
\end{tabular}


From the above Table- 2 it has been observed that, at the end of simulation there are alive nodes in the network whereas in DEEC all the nodes die. In RCEEC, time taken by the first and the tenth node to die in the network increases by approximately 300 rounds.

In addition, RCEEC attains a decrease in the number of packets that are transmitted to CHs. This in turn enhanced network lifetime and increases sustainability of the network.

In the proposed RCEEC protocol results show that No. of packets sent to CHs reduce as compared to DEEC as shown in Figure (1).This results in energy conservation of non-participating (idle) nodes. Total energy of the network increases giving rise to greater number of alive nodes ( Fig. 2) and less number of dead nodes (Fig. 3) per round.

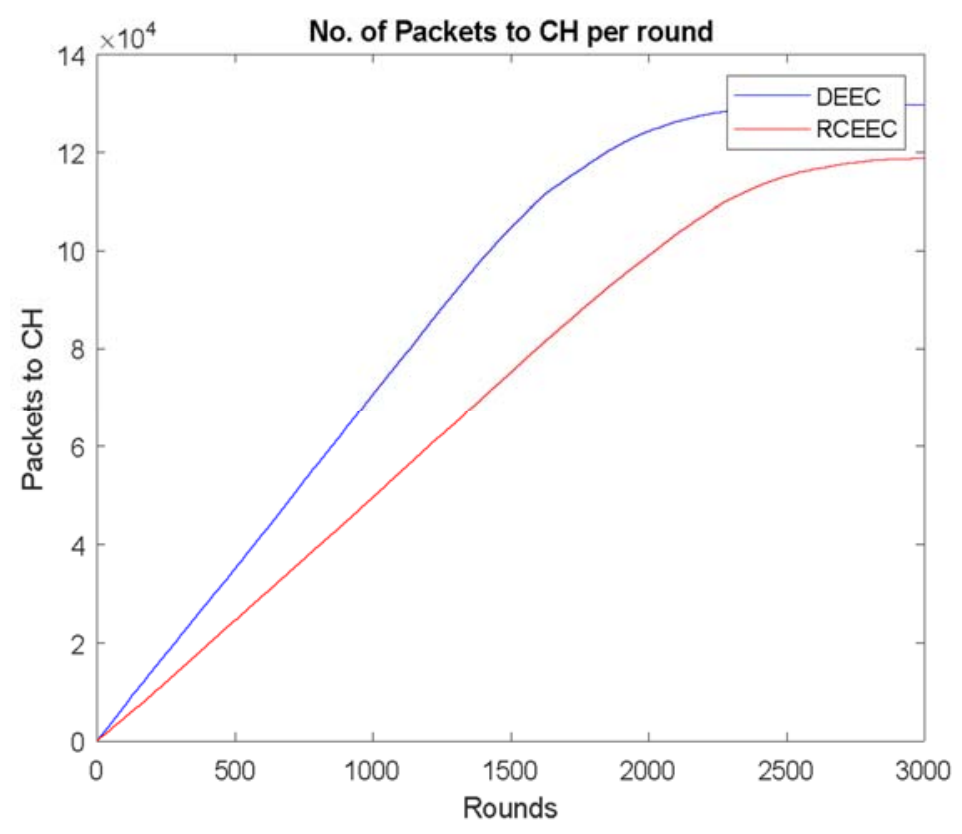

Fig. :-1 No. of packets to $\mathrm{CH}$ per round

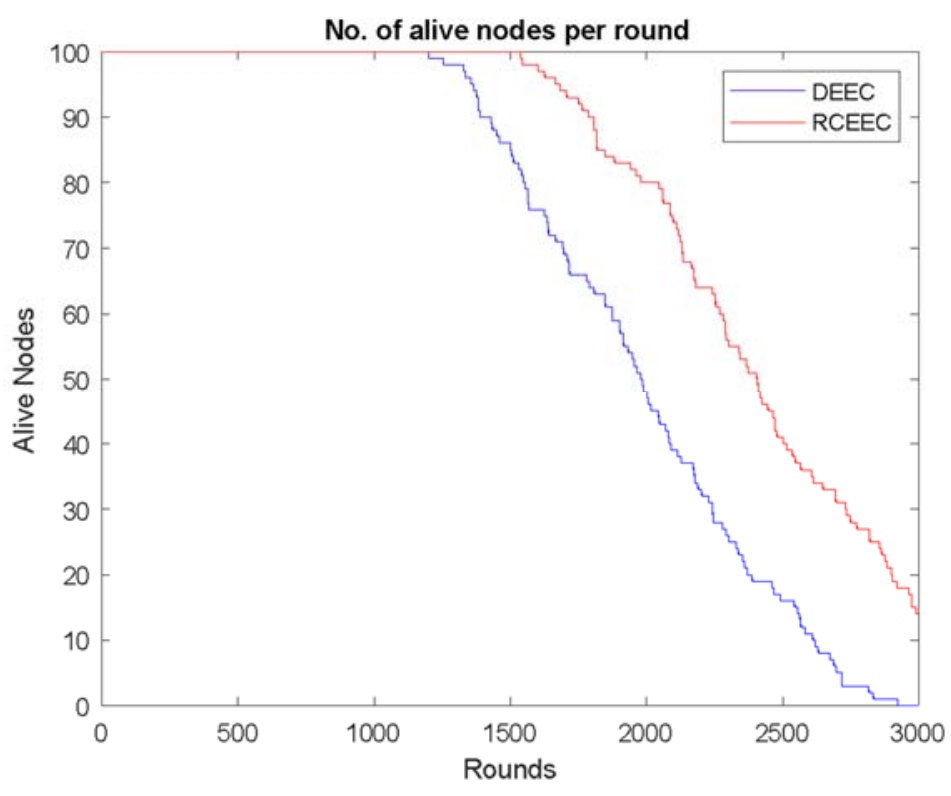

Fig. :-2 No. of alive nodes per round 


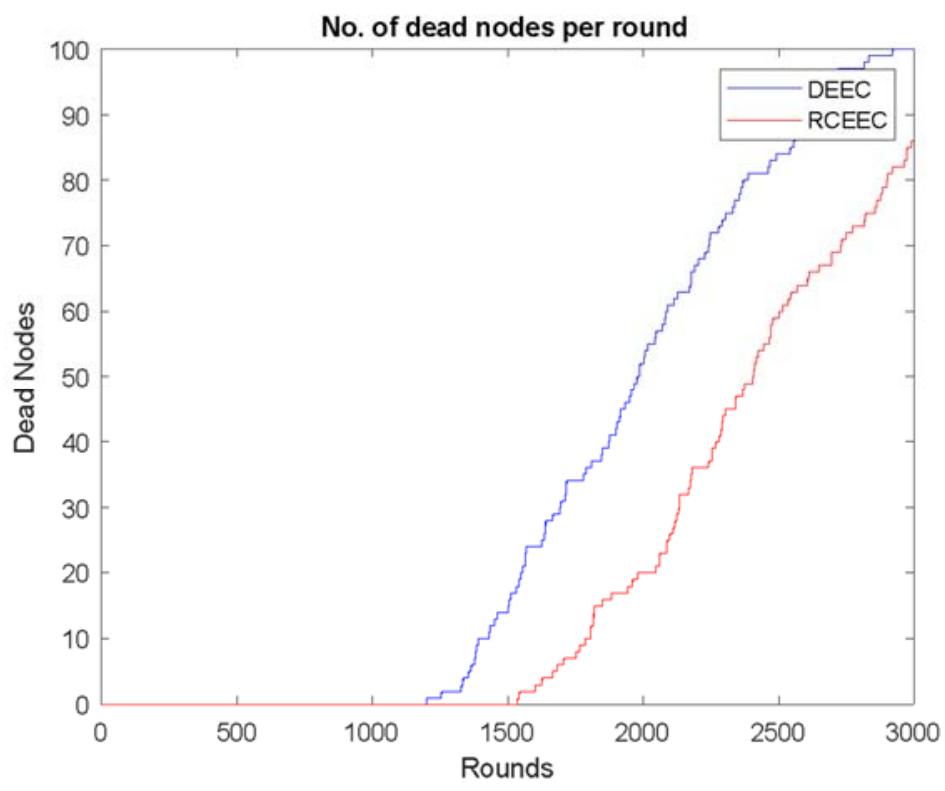

Fig. :-3 No. of dead nodes per round

In this approach numbers of CHs are kept consistent as in the earlier works [2],[4]. There is no significant change in the number of packets sent to the BS as this approach concentrates on reducing only intra cluster communication. Though in RCEEC a slight increase is shown in the number of packets to BS, it can be attributed to longer network lifetime. The stability period (Time lapse between the start of execution and the exhaustion of the first alive node in the WSN) of the network has increased by approximately 300 rounds.

\section{Conclusion}

Smart people living in smart cities are surrounded by innumerous tiny IoT devices that work together to form heterogeneous wireless sensor networks sharing vast array of trivial and non-trivial information at the tips of their fingers. It gives rise to the problem of Big Data lying untouched and consuming important resources. In clustering protocols there are redundancies at two levels- Inter Cluster and Intra Cluster. This protocol RCEEC addresses the problem of intra cluster communication by controlling the transmission packets from nodes to $\mathrm{CH}$. It in turn reduces the amount of data transmitted in the whole sensor network. Simulation results show that it reduces the number of packets in transit saves energy, enhance network lifetime and make the network resilient. RCEEC attains decease in number of packets that are transmitted to CHs. This in turn enhanced network lifetime and increases sustainability of the network. Results show that RCEEC surpasses its predecessor DEEC with reference to energy efficiency, network lifetime and controlling intra cluster traffic.

\section{References}

[1] A. Garg and M. Jailia, "Mining Iot Data for Next Generation Smart Cities", International Journal of Engineering and Advanced Technology, vol. 8, no. 6, pp. 259-262, 2019.

[2] W. Heinzelman, A. Chandrakasan, and H. Balakrishnan, "Energy-efficient routing protocols for wireless microsensor networks," in Proc. 33rd Hawaii Int. Conf. System Sciences (HICSS), Maui, HI, Jan. 2000.

[3] I. Matta, G. Smaragdakis, and A. Bestavros, "SEP: A stable election protocol for clustered heterogeneous wireless sensor networks," in SANPA, August 2004

[4] L. Qing, Q. Zhu, and M. Wang, "Design of a distributed energy-efficient clustering algorithm for heterogeneous wireless sensor networks," Computer Communication, vol. 29, pp. 2230-2237, 2006.

[5] Parul Saini, Ajay.K.Sharma, "E-DEEC- Enhanced Distributed Energy Efficient Clustering Scheme for heterogeneous WSN", in: 2010 1st International Conference on Parallel, Distributed and Grid Computing (PDGC - 2010).

[6] Jibreel, F., "Improved Enhanced Distributed Energy Efficient Clustering (iE-DEEC) Scheme for heterogeneous Wireless Sensor Network", International Journal of Engineering Research and Advanced Technology, vol. 05, no. 01, pp. 06-11, 2019.

[7] Elbhiri, B. , Saadane, R. , El Fkihi, S., Aboutajdine, D. "Developed Distributed Energy-Efficient Clustering (DDEEC) for heterogeneous wireless sensor networks", in: 5th International Symposium on I/V Communications and Mobile Network (ISVC), 2010.

[8] A. Garg and M. Jailia, "Addressing Data Redundancy in IoT Networks", International Journal of Recent Technology and Engineering, vol. 8, no. 4, pp. 8000-8004, 2019.

[9] D. Goldsmith and J. Brusey, The Spanish inquisition protocol—Model based transmission reduction for wireless sensor networks, " in Proc. IEEE Sensors, Nov. 2010, pp. 2043-2048.

[10] E. I. Gaura, J. Brusey, and R. Wilkins, Bare necessities—Knowledgedriven WSN design,“ in Proc. IEEE SENSORS, Oct. 2011, pp. $66-70$.

[11] S. Bhattacharya, T. Blumensath, B. Mulgrew, and M. Davis, -Fast encoding of synthetic aperture radar raw data using compressed sensing, $\|$ in Proc. IEEE Workshop on SSP, Aug. 26-29, 2007, pp. 448-452.

[12] J. Low, A. Azween, "Wireless Sensor Networks: Data Packet Size Optimization”,Universiti Teknologi PETRONAS, Malaysia, 2012.

[13] W. Heinzelman, A. Chandrakasan, and H. Balakrishnan, "An Application-Specific Protocol Architecture for Wireless Microsensor Networks," IEEE Transactions on Wireless Communications, vol. 1, no. 4, pp. 660-670, October 2002. 
[14] M. O. Farooq, A. B. Dogar, and G. A. Shah, "MR-Leach: Multi-hop routing with low energy adaptive clustering hierarchy," in Proc. 4th Int. Conf. Sensor Technol. Appl. (SENSORCOMM), Jul. 2010, pp. 262-268.

[15] K. Maraiya, K. Kant, and N. Gupta, "Efficient cluster head selection scheme for data aggregation in wireless sensor network," Int. J. Comput. Appl., vol. 23, no. 9, pp. 10-18, 2011.

[16] Kumar, S., Verma, S.K., Kumar, A.: 'Enhanced threshold sensitive stable election protocol for heterogeneous wireless sensor network', Wirel. Pers. Commun., 2015, 85, (4), pp. 2643-2656.

[17] K. Shekhar, S. K. Verma, and A. Kumar, "Enhanced threshold sensitive stable election protocol for heterogeneous wireless sensor network," Wireless Pers. Commun., vol. 85, no. 4, pp. 2643-2656, 2015.

[18] A. Malik and S. Singh "hetSEP: Heterogeneous SEP Protocol for Increasing Lifetime in WSNs", Journal of Information and Optimization Sciences, vol. 38, no. 5, pp. 721-743, 2017.

[19] A. Manjeshwar and D. P. Agarwal, "TEEN: a Routing Protocol for Enhanced Efficiency in Wireless Sensor Networks," 1st Int'l. Wksp. on Parallel and Distrib. Comp. Issues in Wireless Networks and Mobile Comp., April 2000.

[20] A. Manjeshwar, D. Agrawal, "APTEEN: A Hybrid Protocol for Efficient Routing and Comprehensive Information Retrieval in Wireless Sensor Networks," In Proc. International Parallel and Distributed Processing Symposium, Florida, 2002, pp. 195-202.

[21] J. Ma, S. Wang, and Y. Ge, "Ant-Colony Based Double Cluster Heads Adaptive Periodic Threshold-Sensitive Energy Efficient Network Protocol in WSN," Lecture Notes in Electrical Engineering Communications, Signal Processing, and Systems, pp. 309-317, 2017.

[22] J. Ma, S. Wang, C. Meng, Y. Ge, and J. Du, "Hybrid energy-efficient APTEEN protocol based on ant colony algorithm in wireless sensor network,” EURASIP J. Wireless Commun. Netw., vol. 102, pp. 1-13, Dec. 2018. 